\title{
Altered microRNA expression in cisplatin-resistant ovarian cancer cells and upregulation of miR-130a associated with MDR1/P-glycoprotein-mediated drug resistance
}

\author{
LINGYUN YANG, NINGWEI LI, HONGJING WANG, XIBIAO JIA, XUE WANG and JUAN LUO \\ Department of Gynecology and Obstetrics, West China Second University Hospital, \\ Sichuan University, Chengdu, Sichuan 610041, P.R. China
}

Received February 7, 2012; Accepted April 2, 2012

DOI: $10.3892 /$ or.2012.1823

\begin{abstract}
RNAs (miRNAs) are short non-coding RNA molecules which are involved in the regulation of various biological processes. Drug resistance has become a major obstacle to successful chemotherapy of ovarian cancer. The aim of this study was to investigate microRNA expression profiles in cisplatin-resistant ovarian cancer cells and the role of miR-130a in regulating drug resistance. Analysis of differentially expressed miRNAs between SKOV3 and SKOV3/CIS cells was assessed by miRNA microarrays. Target prediction of miRNAs was determined with the help of PicTar or TargetScan. Among these miRNAs, the expression of miR-130a was verified using qRT-PCR. The expression of MDR1 mRNA and P-glycoprotein (P-gp) after cellular transfection was examined using qRT-PCR and western blotting, respectively. Cisplatin sensitivity was detected by the MTT assay. We indentified 35 downregulated and 54 upregulated miRNAs in SKOV3/CIS compared to those in SKOV3. We found that miR-130a was upregulated in SKOV3/CIS compared to the parental SKOV3 cells, and PTEN was predicted to be the potential target of miR-130a. Moreover, downregulation of miR-130a could inhibit MDR1 mRNA and P-gp expression and overcome the cisplatin resistance in SKOV3/CIS cells, which indicated that miR-130a may be associated with MDR1/P-gp-mediated drug resistance and plays the role of an intermediate in drug-resistance pathways of PI3K/Akt/PTEN/ mTOR and ABC superfamily drug transporters in SKOV3/ CIS cells. This study provides important information for the
\end{abstract}

Correspondence to: Professor Hongjing Wang, Department of Gynecology and Obstetrics, West China Second University Hospital, Sichuan University, Renmin South Road Section 3 No. 17, Chengdu, Sichuan 610041, P.R. China

E-mail:whjscdx@163.com

Key words: ovarian cancer, cisplatin resistance, microRNA, expression profiling, $\mathrm{miR}-130 \mathrm{a}$ development of targeted gene therapy for reversing cisplatin resistance in ovarian cancer.

\section{Introduction}

Ovarian cancer is the deadliest cancer of the female reproductive system (1). More than $70 \%$ newly diagnosed patients with ovarian cancer have advance stage disease (FIGO stage III or IV) because of no early symptoms and effective biomarker screening (2). Primary cytoreduction followed by platinumbased combined chemotherapy, such as cisplatin coupled with paclitaxel, has become the main therapeutic approach for advanced ovarian cancer (3-6). Cisplatin crosslinks to double stranded DNA and forms DNA adducts, interfering with DNA replication and transcription, which in turn activates cellular apoptosis. However, development of a multidrug resistance phenotype is a major obstacle to successful treatment of ovarian cancer, which leads to $85-90 \%$ relapse and only $30 \%$ 5 -year survival rate $(6,7)$. Several molecular mechanisms of drug resistance have been discovered and characterized including the overexpression of MDR1/P-glycoprotein (P-gp) $(8,9)$, increased anti-apoptotic factors activity $(8,10,11)$, altered expression of $\beta$-tubulin subunits (12), degeneration of growth factor receptor (13), and increased DNA repair activity $(14,15)$. Drug efflux induced by increased accumulation of various ATP-binding cassette (ABC) transportors, including ABCB1, also known as P-gp, which is a $170-\mathrm{kDa}$ transmembrane glycoprotein encoded by MDR1 gene on human chromosome $7 \mathrm{p} 21$, has been demonstrated to play a significant role in drug resistance $(8,9,16)$.

microRNAs (miRNAs) are a class of short non-coding RNA molecules that bind to the 3'-untranslated region (3'-UTR) of the target mRNAs and act post-transcriptionally as negative regulators of gene expression (17-19). Mature miRNAs, derived from precursor miRNA (pre-miRNA), are incorporated into the RNA-induced silencing complex (RISC), resulting in either cleavage or translational repression of target mRNAs $(20,21)$. It has been reported that miRNAmediated gene regulation plays critical roles in biological processes including cellular proliferation, differentiation, apoptosis, metabolism and oncogenesis $(17,22,23)$. Recent studies show that miRNAs associated with oncogenesis act 
as oncogenes or tumor suppressors. For example, miR-155, upregulated in ovarian and lung cancer, acts as an oncogene $(24,25)$, while miR-15a and miR-16a, deregulated in CLL, act as tumor suppressors (26). In addition, further reports show that miRNAs also play a vital role in chemotherapeutic drug resistance (27-29). Analysis of the up- and downregulation and the mechanism of action of miRNAs may be useful to develop novel plans of targeted therapies.

In the current study, we performed miRNAs expression profile analysis between the human ovarian cancer cell line SKOV3 and the cisplatin-resistant cell line SKOV3/CIS followed by qRT-PCT based validation of miR-130a expression. We also examined the role of miR-130a in the development of cisplatin-resistance in ovarian cancer cells, which may provide a potential target for gene therapy.

\section{Materials and methods}

Cell culture. The human ovarian cancer cell line SKOV3 and the cisplatin-resistant cell line SKOV3/CIS were cultured in PRMI-1640 medium (Gibco, Carlsbad, CA, USA) supplemented with $10 \%$ fetal bovine serum (FBS, Gibco), $100 \mathrm{U} /$ $\mathrm{ml}$ penicillin and $100 \mu \mathrm{g} / \mathrm{ml}$ streptomycin in a humidified incubator with $5 \% \mathrm{CO}_{2}$ at $37^{\circ} \mathrm{C}$. SKOV3/CIS was alternately fed with medium containing $7.5 \mu \mathrm{g} / \mathrm{ml}$ cisplatin and was regularly tested for maintenance of drug-resistance. Growth and morphology of each cell line were observed and monitored every three days. The cisplatin-resistant cell was maintained in drug-free medium for 1 week before follow-up experiments. Both cell lines were preserved in Gynecological Oncology of Biotherapy Laboratory, Department of Gynecology and Obstetrics, West China Second University Hospital, Sichuan University, Chengdu, Sichuan, China.

miRNA microarray analysis. Total RNA was isolated using TRIzol reagent (Invitrogen, Carlsbad, CA, USA) and the miRNeasy mini kit (Qiagen, Copenhagen, Denmark) according to the manufacturer's instructions, which efficiently covered all RNA species including miRNAs. RNA quality and quantity was measured using the NanoDrop spectrophotometer (ND-1000, NanoDrop Technologies) and RNA integrity was determined by gel electrophoresis. The miRCURY ${ }^{\mathrm{TM}}$ Power labeling kit (Exiqon, Vedbaek, Denmark) was used for miRNA labeling. Each sample $(1 \mu \mathrm{g})$ was 3'-end-labeled with $\mathrm{Hy}^{\mathrm{TM}}$ fluorescent label using T4 RNA ligase. Then the Hy $3^{\mathrm{TM}}$-labeled samples were hybridized on the miRCURY ${ }^{\mathrm{TM}}$ LNA Array (v.16.0, Exiqon), which contained more than 1,891 capture probes, covering all human, mouse and rat miRNAs annotated in miRBase 16.0, as well as all viral miRNAs related to these species. In addition, this array contained capture probes for 66 new miRPlus ${ }^{\mathrm{TM}}$ human miRNAs which were proprietary miRNAs not found in miRBase. The total $25-\mu 1$ mixture from $\mathrm{Hy} 3^{\mathrm{TM}}$-labeled samples with $25 \mu \mathrm{l}$ hybridization buffer were first denatured for $2 \mathrm{~min}$ at $95^{\circ} \mathrm{C}$, incubated on ice for $2 \mathrm{~min}$ and then hybridized to the microarray for 16-20 h at $56^{\circ} \mathrm{C}$ in a 12 -Bay hybridization systems (NimbleGen Systems, Inc., Madison, WI, USA). Following hybridization, the slides were washed, dried and then scanned using the Axon GenePix 4000B microarray scanner (Axon Instruments, Foster City, CA, USA). Images were then imported into GenePix Pro
6.0 software (Axon) for grid alignment and data extraction. Expressed data were normalized using the median normalization. The normalized fluorescence intensity, which was less than the average intensity of the negative controls or 3x SD of the intensity of the negative controls, indicated an unreliable signal value. After normalization, differentially expressed miRNAs were identified through fold-change filtering. Hierarchical clustering was performed using standard correlation as a measure of similarity by the MEV software (v4.6, TIGR). Three microarray chips were applied for each cell line.

Bioinformatics. Target prediction of differentially expressed miRNAs was determined with the help of PicTar (http:// pictar.mdc-berlin.de/) $(30,31)$ or TargetScan 5.2 (http://www. targetscan.org/) $(32,33)$.

Real-time $q R T$-PCR for miR-130a. Verification of altered miR-130a expression was performed in SKOV3 and SKOV3/ CIS by real-time qRT-PCR. Total RNA of each cell line was extracted with TRIzol reagent (Invitrogen) and was assessed by agarose gel electrophoresis and spectrophotometry. Complementary DNA (cDNA) was generated using the AMV first strand cDNA synthesis kit (Invitrogen). Specific miR-130a primers and internal control U6 snRNA primers (Bulge-LoopT ${ }^{\mathrm{TM}}$ miRNA qPCR Primers) were designed and synthesized by Guangzhou RiboBio (Guangzhou RiboBio Co., Ltd., Guangzhou, China). Real-time qRT-PCR was performed in a $20-\mu 1$ reaction volume containing $2 \mu \mathrm{l}$ cDNA template, $9 \mu \mathrm{l}$ SYBR-Green I mix (Takara, China), $2 \mu$ l forward primers, $2 \mu \mathrm{l}$ reverse primer and $5 \mu \mathrm{l}$ RNase-free $\mathrm{H}_{2} \mathrm{O}$ on the ABI StepOnePlus instrument (Applied Biosystems, USA) using the following protocol: $95^{\circ} \mathrm{C}$ for $20 \mathrm{sec}$, followed by 40 cycles of $95^{\circ} \mathrm{C}$ for $10 \mathrm{sec}, 60^{\circ} \mathrm{C}$ for $20 \mathrm{sec}$ and $70^{\circ} \mathrm{C}$ for $10 \mathrm{sec}$. Each sample was run in triplicate. The relative expression of miR$130 \mathrm{a}$ was analyzed using $2^{-\Delta \Delta \mathrm{Ct}}$ method.

miRNA transfection. The miR-130a-mimic, -inhibitor and miR-negative RNA were designed and chemically synthesized by Guangzhou RiboBio. Twenty-four hours prior to transfection, SKOV3 and SKOV3/CIS cells were seeded into 6-well plates with $2 \times 10^{5}$ cells/well and cultured in medium without antibiotics. Transfection was performed using Lipofectamine 2000 (Invitrogen) and OPTI-MEM I reduced serum medium (Gibco) according to the manufacturer's instructions. Green fluorescent protein (GFP) was applied to show the efficiency of transfection system. Cells in each well were transfected with $10 \mu 1 \mathrm{miR}-130 \mathrm{a}-\mathrm{mimic}$ or -inhibitor or miR-negative RNA or $2 \mu \mathrm{l}$ GFP constructed plasmid. The medium was replaced 4-6 $\mathrm{h}$ after transfection with new fresh medium.

Real-time qRT-PCR for MDR1 mRNA. Real-time qRT-PCR was applied to detect the expression of MDR1 mRNA after miRNA transfection for $24 \mathrm{~h}$. Total RNA of each cell line was extracted with TRIzol reagent (Invitrogen) and was assessed by agarose gel electrophoresis and spectrophotometry. Reverse transcription was carried out with the AMV first strand cDNA synthesis kit. The $\beta$-actin was used as internal control for MDR1 expression normalization. Primers of MDR1 and $\beta$-actin were designed using the Primer Premier software (version 5.0). The primer sequences for real-time qRT-PCR 
were: MDR1 (forward) 5'-ACAAAGTGCTCACCCAGA TTC-3' (reverse) 5'-CATTTCCTCGCTGATGACACTA-3'; $\beta$-actin (forward) 5'-GCTACGAGCTGCCTGACG-3' (reverse) 5'-TCGTGGATGCCACAGGAC-3'. Real-time qRT-PCR was performed with cycle parameters as $95^{\circ} \mathrm{C}$ for $10 \mathrm{~min}$, followed by 40 cycles of $95^{\circ} \mathrm{C}$ for $15 \mathrm{sec}, 60^{\circ} \mathrm{C}$ for $1 \mathrm{~min}$. Each sample was run in triplicate. Data analysis was performed using the $2^{-\Delta \Delta \mathrm{Ct}}$ method.

Western blot analysis. Cells were collected and washed twice with ice-cold PBS. Total protein was extracted with RIPA added with $1 \%$ protease inhibitor phenylmethanesulfonyl fluoride (PMSF) for $15 \mathrm{~min}$ on ice, then centrifuged at $12,000 \mathrm{rpm} 4^{\circ} \mathrm{C}$ for $15 \mathrm{~min}$ and the supernatants were collected. Protein concentrations were determined by the BCA method using the standard curve. GAPDH expression was used as internal control. Equal amounts of protein for each sample were resolved using 10\% SDS-PAGE gels and transferred onto Immun-Blot ${ }^{\mathrm{TM}}$ PVDF membranes (Bio-Rad Laboratories, Hercules, CA, USA). The bands were blocked with a 5\% solution of skim dry milk in TBS containing $0.1 \%$ Tween-20 for $2 \mathrm{~h}$ and incubated with primary antibodies P-gp (diluted 1:1000; Calbiochem, San Diego, CA, USA) and GAPDH (diluted 1:50000; Kangchen, Shanghai, China) overnight at $4^{\circ} \mathrm{C}$. After repeated washing three times with TBS-T, membranes were incubated with horseradish peroxidase (HRP) conjugated anti-mouse secondary antibody (Bio-Rad Laboratories) for $2 \mathrm{~h}$. Blots were detected and developed on $\mathrm{X}$-ray film using the chemiluminescence method.

In vitro drug sensitivity array. SKOV3 and SKOV3/CIS cells were transfected with miR-130a-mimic or -inhibitor or miRnegative RNA for $24 \mathrm{~h}$, and then transferred into 96-well plates with $1.5 \times 10^{4}$ cells/well for a drug sensitivity array. After cellular adhesion, SKOV3 cells were treated with increasing concentrations of cisplatin $(0.3,0.6,1.2,2.4,4.8$ and $9.6 \mu \mathrm{g} / \mathrm{ml})$ and SKOV3/CIS were exposed to various doses of cisplatin (3, $6,12,24,48$ and $96 \mu \mathrm{g} / \mathrm{ml}$ ) for $48 \mathrm{~h}$. Following the incubation, $20 \mu \mathrm{l}$ of $5 \mathrm{mg} / \mathrm{ml} \mathrm{3-(4,5-dimethylthiazol-2-yl)-2,5-diphenyl-}$ tetrazolium bromide (MTT, Sigma, St. Louis, MO, USA) was added to each well and incubated for $4 \mathrm{~h}$. At the end of the incubation, the medium was removed and $150 \mu 1$ DMSO was added. Absorbance of each well was measured at a wavelength of $570 \mathrm{~nm}$ using a microplate reader (Model 680, Bio-Rad Laboratories). The $\mathrm{IC}_{50}$ median inhibitory concentration of cisplatin was calculated through a cell viability curve.

Data and statistical analysis. Data are presented as the means \pm standard deviation from at least three replicate experiments. One-way ANOVA was employed to analyze difference of means between groups using SPSS 11.0 software (SPSS Inc., Chicago, IL, USA). Statistical significance was set as $\mathrm{P}<0.05$.

\section{Results}

Profiling of differentially expressed miRNAs in SKOV3 and SKOV3/CIS cells. RNA quantity and quality of each cell line were assured and checked by spectrophotometer and gel electrophoresis. As shown in Table I, the results confirmed good
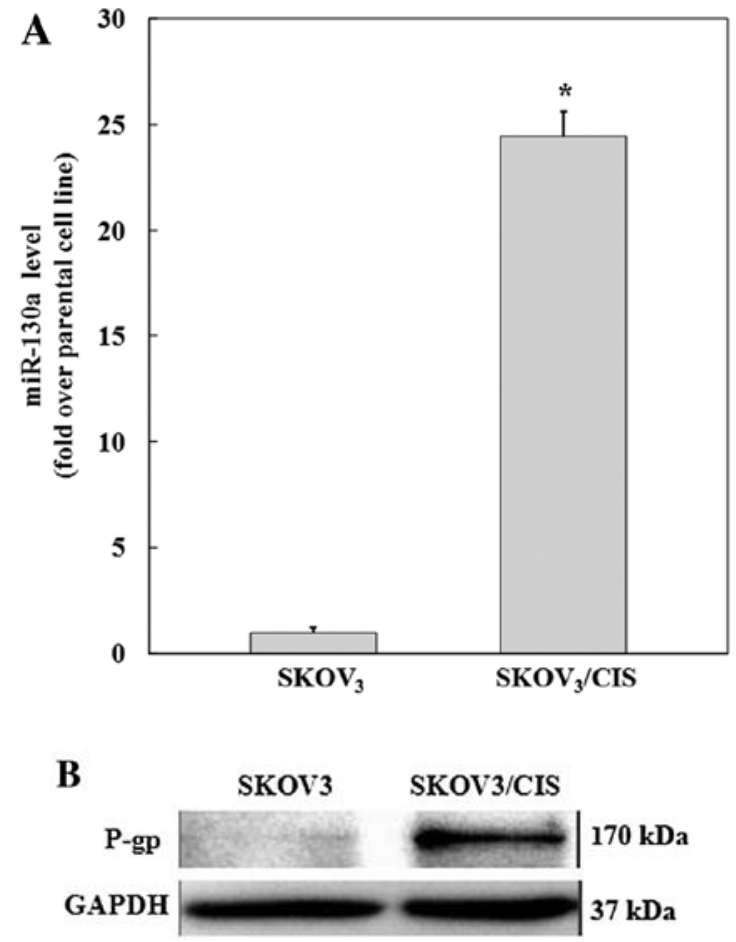

Figure 1. Expression of miR-130a and P-gp in SKOV3 and SKOV3/CIS cell lines. (A) The mean fold-change of miR-130a expression was detected by qRT-PCR in SKOV3 and SKOV3/CIS. The relative expression of miR-130a in SKOV3/CIS cells was 24.43-fold higher than that in SKOV3 cells, ${ }^{*} \mathrm{P}<0.05$ Each sample was analyzed in triplicate and normalized to U6 snRNA. Foldchange was figured out by $2^{-\Delta \Delta C t}$ method. (B) The expression of P-gp, as measured using western blotting, was upregulated in SKOV3/CIS and was absent in SKOV3. GAPDH was used as internal control.

quantity and quality of total RNA isolated from each cell line. To identify the critical miRNAs involved in cisplatin resistance, the microarray platform was applied to profile the global expression of mature miRNA. The signal ratio of SKOV3/CIS to SKOV3 wasdetermined, and the normalized fluorescence intensity, which was less than the average intensity of the negative controls or 3x SD of the intensity of the negative controls indicated an unreliable signal value. Differentially expressed miRNAs with statistical significance were selected only when they were altered by at least 2 -fold. With these strict criteria, 35 miRNAs exhibited lower expression and 54 miRNAs exhibited higher expression in SKOV3/CIS than those in SKOV3 cells (Tables II and III). Expression of miRNAs in SKOV3/CIS compared to SKOV3 was decreased by 2.02- to 8.23-fold, while increases in expression ranged from 2.02- to 46.74-fold. These results indicated that such differentially expressed miRNAs might play a crucial role in the development of cisplatin resistance in epithelial ovarian cancer. The tumor suppressor PTEN was predicted to be a potential target of miR-130a.

Expression of miR-130a and P-gp in SKOV3 and SKOV3/ $C I S$. We performed real-time qRT-PCR for further validation of miR-130a expression level and western blot analysis for comparison of P-gp expression in SKOV3 and SKOV3/CIS cell lines, respectively. High values of relative threshold cycles indicate the low miRNA quantity, which were normalized by U6 snRNA expression, and GAPDH was used as internal 
Table I. RNA quantity and quality of each cell line assessed by spectrophotometry.

\begin{tabular}{lcccc}
\hline Cell line & A260/A280 ratio & A260/A230 ratio & Total RNA concentration (ng/ $\mu$ l) & Total RNA amount (ng) \\
\hline SKOV3 & 2.01 & 2.22 & 1574.25 & 15742.5 \\
SKOV3/CIS & 2.00 & 2.35 & 908.09 & 9080.9
\end{tabular}

For spectrophotometry, the A260/A280 ratio should be close to 2.0 for pure RNA (ratios between 1.8 and 2.1 are acceptable) and the A260/ A230 ratio should be $>1.8$, which indicates good quantity and quality of total RNA.

Table II. Thirty-five downregulated miRNAs in SKOV3/CIS cell lines and the putative targets.

\begin{tabular}{|c|c|c|c|}
\hline miRNA ID & Accession no. & Fold-change & Predicted targets \\
\hline hsa-let-7i* & MIMAT0004585 & 0.1215 & Unknown \\
\hline hsa-miR-9* & MIMAT0000442 & 0.1520 & Unknown \\
\hline hsa-miR-630 & MIMAT0003299 & 0.1534 & TMED7, FLJ36031, LMO3, TOB2, TFAP2B \\
\hline hsa-miR-21* & MIMAT0004494 & 0.1658 & Unknown \\
\hline hsa-miR-205* & MIMAT0009197 & 0.1715 & Unknown \\
\hline hsa-miR-498 & MIMAT0002824 & 0.2173 & TIMM17A, TACSTD1, GRIA3, CREBL2, TP53INP1, ATP2B1 \\
\hline hsa-miR-31 & MIMAT0000089 & 0.2509 & RSBN1, PIK3C2A, SLC1A2, PRKCE \\
\hline hsa-miRPlus-J1011 & Unknown & 0.2599 & Unknown \\
\hline hsa-miR-210 & MIMAT0000267 & 0.2768 & GIT2, ZNF462, FAM116A, KCMF1 \\
\hline hsa-miR-584 & MIMAT0003249 & 0.2826 & PRRX1, CADPS, STYX, PHACTR1 \\
\hline hsa-miR-1265 & MIMAT0005918 & 0.2926 & BCL-2, IRF2, XPO5 \\
\hline hsa-miR-4308 & MIMAT0016861 & 0.2964 & Unknown \\
\hline hsa-miR-1264 & MIMAT0005791 & 0.2980 & ATP2C1, ABCA1, BRCC3, CS, KCNG3, LRRC18 \\
\hline hsa-miR-4324 & MIMAT0016876 & 0.2984 & Unknown \\
\hline hsa-miR-375 & MIMAT0000728 & 0.3045 & ITPKB, RASD1, PTPN4, ATP2B3 \\
\hline hsa-miR-3074-3p & MIMAT0015027 & 0.3345 & Unknown \\
\hline hsa-let-7a-2* & MIMAT0010195 & 0.3603 & Unknown \\
\hline hsa-miR-208a & MIMAT0000241 & 0.3711 & CHD9, EIF4G2, PDCD4, FNIP1, FNIP2 \\
\hline hsa-miR-2116 & MIMAT0011160 & 0.3798 & Unknown \\
\hline hsa-miR-1321 & MIMAT0005952 & 0.3845 & KLK4, RASSF5, ABCG4, MTA2 \\
\hline hsa-miR-668 & MIMAT0003881 & 0.3872 & SKAP2, ATP6AP2, HOXB4, ID4, TOX3, SLC26A7 \\
\hline hsa-miR-1913 & MIMAT0007888 & 0.3945 & Unknown \\
\hline hsa-miR-513a-5p & MIMAT0002877 & 0.4201 & CSNK1G1, GLT8D3, EPS8, TNPO1 \\
\hline hsa-miR-24-1* & MIMAT0000079 & 0.4274 & Unknown \\
\hline hsa-miR-2115* & MIMAT0011159 & 0.4403 & Unknown \\
\hline hsa-miR-3686 & MIMAT0018114 & 0.4413 & Unknown \\
\hline hsa-miR-1973 & MIMAT0009448 & 0.4434 & Unknown \\
\hline hsa-miR-1284 & MIMAT0005941 & 0.4463 & TADA1L, MARK3, MARK1, ATP2B2 \\
\hline hsa-miR-3182 & MIMAT0015062 & 0.4620 & Unknown \\
\hline hsa-miR-193b* & MIMAT0004767 & 0.4780 & Unknown \\
\hline hsa-miR-3667-5p & MIMAT0018089 & 0.4809 & Unknown \\
\hline hsa-miR-181a & MIMAT0000256 & 0.4831 & PIP3AP, ATP2B2, HOXB5, HOXA11, ATP2A2 \\
\hline hsa-miR-3926 & MIMAT0018201 & 0.4907 & Unknown \\
\hline hsa-miR-664 & MIMAT0005949 & 0.4917 & HS6ST3, TNFAIP1, CYBB, GPR180 \\
\hline hsa-miR-615-3p & MIMAT0003283 & 0.4942 & C6orf154, VSTM2L, GRIA4, SHANK3 \\
\hline
\end{tabular}

Normalized hybridization signal ratio of miRNAs in SKOV3/CIS to those in SKOV3. The normalized fluorescence intensity, which was less than the average intensity of negative controls or 3x SD of the intensity of the negative controls, indicated an unreliable signal value. Differentially expressed miRNAs with statistical significance were selected only if they were altered by at least 2 -fold $(\mathrm{P}<0.05)$. Expression of miRNAs in SKOV3/CIS compared to SKOV3 was decreased by 2.02- to 8.23-fold. Predicted targets are listed with the help of PicTar and TargetScan. 

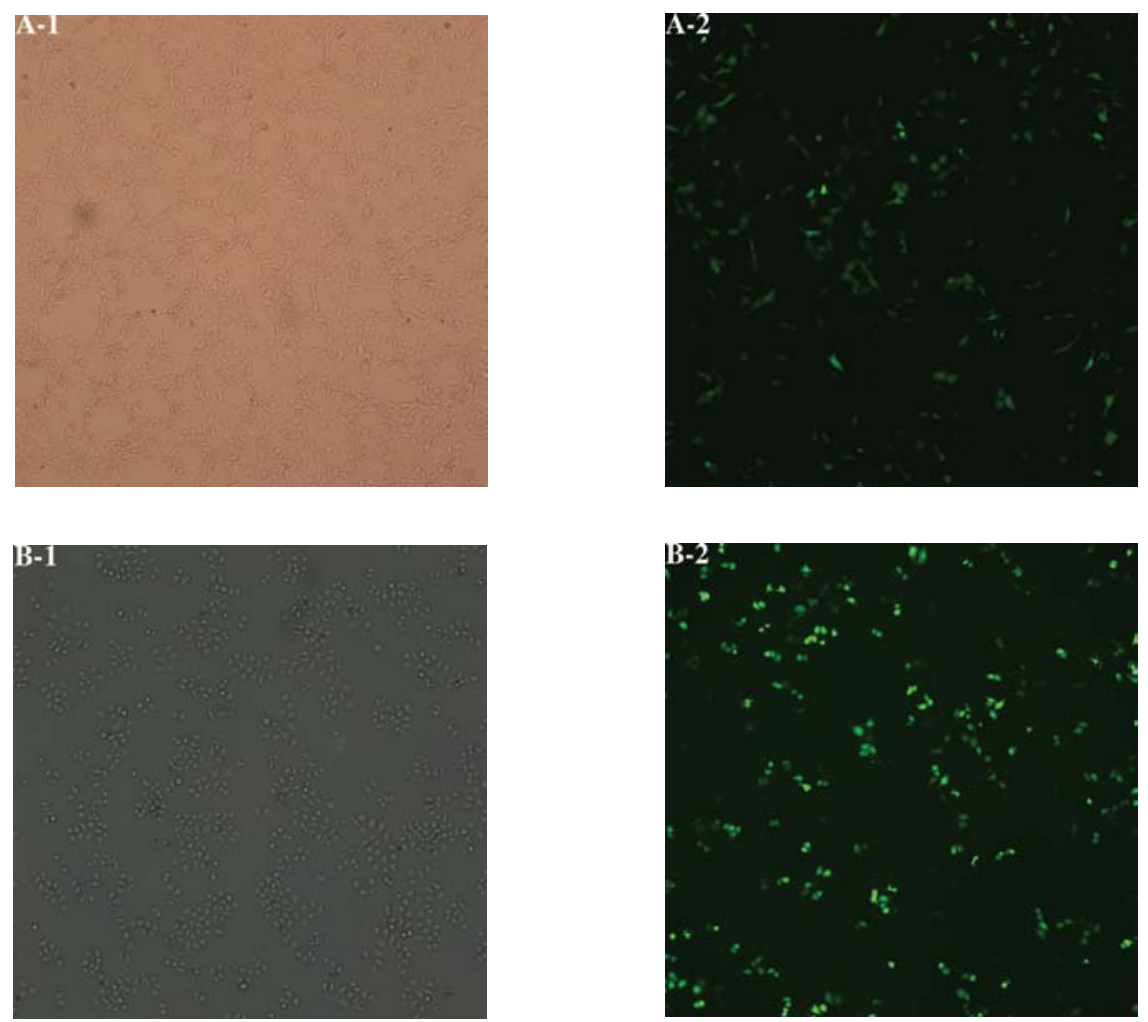

Figure 2. Expression of GFP in SKOV3 and SKOV3/CIS cell lines (original magnification, x40). (A) SKOV3 cells were transfected with the plasmid labeled with GFP gene sequences. GFP expression was observed under a fluorescence microscope after $48 \mathrm{~h}$ of transfection (A-1, normal light; A-2, fluorescent light). (B) After $48 \mathrm{~h}$ of transfection, GFP expression in SKOV3/CIS was observed under a fluorescence microscope (B-1, normal light; B-2, fluorescent light).

control. As shown in Fig. 1A, the expression levels of miR-130a were on average 24.43-fold higher in SKOV3/CIS cell than in parental SKOV3 cell $(\mathrm{P}<0.05)$, which was concordant with the result of miRNA expression profiling. In addition, western blotting showed that the expression level of P-gp was very high in SKOV3/CIS and was absent in SKOV3 cells (Fig. 1B). Overall, these findings suggest that upregulation of miR-130a in SKOV3/CIS was related to MDR1/P-glycoprotein-mediated cisplatin resistance.

Expression of GFP indicating the high efficiency of the liposome-mediated transfection system. We constructed the plasmid labeled with GFP gene sequences to assess the efficiency of the Lipofectamine 2000-mediated transfection system of ovarian cancer cells. After $48 \mathrm{~h}$ of transfection, microphotographs of both cell lines were captured under a fluorescence microscope (Nikon Eclipse 80I, Japan). As presented in Fig. 2, nearly $80 \%$ of green fluorescence could be clearly observed and detected in the cytoplasm of each cell line, which indicated that the liposome-mediated transfection system in our experiment, could reach high efficiency.

Transfection of miR-130a modulating MDRI and P-gp expression in SKOV3 and SKOV3/CIS. To further investigate whether miR-130a is associated with MDR1/P-gp-mediated drug resistance, we examined the MDR1 mRNA and P-gp expression using qRT-PCR and western blot analysis after transfection. As shown in Fig. 3, the expression of MDR1 mRNA was upregulated in cisplatin sensitive SKOV3 cells transfected with an miR-130a-mimic, while inhibiting miR- 130a expression could remarkably decreased MDR1 and $\mathrm{P}$-gp expression in drug resistant SKOV3/CIS cell, compared to miR-negative control $(\mathrm{P}<0.05)$. Overall, these results might indicate that upregulation of miR-130a in ovarian cancer cells is likely associated with MDR1/P-glycoproteinmediated drug resistance.

Transfection of miR-130a regulating cisplatin sensitivity in SKOV3 and SKOV3/CIS cells. In vitro drug sensitivity array analysis was carried out to validate the role of miR-130a in cisplatin resistance of ovarian cancer cells. After transfection with a miR-130a-mimic or -inhibitor or with miR-negative, SKOV 3 and SKOV3/CIS cells were treated with increasing doses of cisplatin. Cell viability curves are shown in Fig. 4, through which the $\mathrm{IC}_{50}$ of each sample was calculated. We found that cisplatin sensitivity was decreased in SKOV3 cells transfected with the miR-130a-mimic, while downregulating miR-130a expression overcame cisplatin resistance in SKOV3/ CIS cells.

\section{Discussion}

Chemotherapy remains an essential component for the treatment of ovarian cancer. Although platinum-based combined therapy has a good initial response, development of multidrug resistance is a major obstacle to successful treatment, which leads to relapse and poor prognosis. Consequently, it is necessary to discover novel targeting specific molecules regulating drug resistance and to develop rational approaches for further therapies. In recent years, accumulating evidence of the role of 
Table III. Fifty-four upregulated miRNAs in SKOV3/CIS cell lines and the putative targets.

\begin{tabular}{|c|c|c|c|}
\hline miRNA ID & Accession no. & $\begin{array}{l}\text { Fold- } \\
\text { change }\end{array}$ & Predicted targets \\
\hline hsa-miR-1290 & MIMAT0005880 & 2.0220 & STK17A, KCNAB1, CDKN2B, STK3, TPRG1, MTMR3 \\
\hline hsa-miR-3651 & MIMAT0018071 & 2.0978 & Unknown \\
\hline hsa-miR-27a & MIMAT0000084 & 2.1093 & PLK2, HIPK2, MAP3K7IP3, SLC6A1 \\
\hline hsa-miR-17 & MIMAT0000070 & 2.1367 & SCN1A, MAP3K2, ITGB8, CASP2, AEN, CASP7 \\
\hline hsa-let-7e & MIMAT0000066 & 2.1426 & HMGA2, CASP3, MAP4K3, CDKNA1, CDCA8 \\
\hline hsa-let-7i & MIMAT0000415 & 2.2700 & CDC34, TTLL4, CDC25A, HOAX1, CASP3 \\
\hline hsa-miR-378 & MIMAT0000732 & 2.3502 & CDC40, IPO9, COAS2, CDC25A, PPIA \\
\hline hsa-miR-4284 & MIMAT0016915 & 2.3612 & Unknown \\
\hline hsa-miR-133b & MIMAT0000770 & 2.3670 & KIF3C, CDC2L5, ABCC1, RICTOR \\
\hline hsa-miR-106a & MIMAT0000103 & 2.3823 & SCN1A, CDKNA1, CASP2, BCL2L11 \\
\hline hsa-miR-148b & MIMAT0000759 & 2.3954 & ATP6AP2, BCL2L11, ABCB7, CDC2L6, CDK5R1 \\
\hline hsa-miR-181d & MIMAT0002821 & 2.5323 & Unknown \\
\hline hsa-miR-29b & MIMAT0000100 & 2.5671 & COL1A1, PTEN, PPIC, CASP7, CDK6 \\
\hline hsa-let-7c & MIMAT0000064 & 2.5717 & HMGA2, CDC34, CDC25A, CASP3 \\
\hline hsa-miR-3175 & MIMAT0015052 & 2.8417 & Unknown \\
\hline hsa-let-7g & MIMAT0000414 & 2.9314 & HIC2, CDC34, TTLL4, CCND2, CDC25A \\
\hline hsa-miR-30b & MIMAT0000420 & 3.0119 & CELSR3, ADRB1 \\
\hline hsa-miR-92a & MIMAT0000092 & 3.1155 & CD69, PIK3R3, AATK, HIPK1, BCL9 \\
\hline hsa-miR-4289 & MIMAT0016920 & 3.1368 & Unknown \\
\hline hsa-miR-23a & MIMAT0000078 & 3.4013 & APAF1, CASP7, MAP4, CDC40 \\
\hline hsa-miR-744 & MIMAT0004945 & 3.5532 & KLC2, LRP3, PPFIA3 \\
\hline hsa-miR-320d & MIMAT0006764 & 3.6503 & CDK6, TRIAP1, CAPRIN1, CDC2L5 \\
\hline hsa-miR-3653 & MIMAT0004955 & 3.6628 & Unknown \\
\hline hsa-miR-320a & MIMAT0000510 & 3.6906 & CDK6, TRIAP1, CAPRIN1, CDC2L5 \\
\hline hsa-miR-99a & MIMAT0000097 & 4.0488 & THAP, TRAF7 \\
\hline hsa-miR-221 & MIMAT0000278 & 4.2337 & STK17B, CDKN1B, COAS2, CDC2L11, ABCC5 \\
\hline hsa-miR-15a & MIMAT0000068 & 4.2364 & BCL2L2, CDC42, ABCC5 \\
\hline hsa-miR-365 & MIMAT0000710 & 4.3053 & SLC30A7, CDC25A \\
\hline hsa-miR-98 & MIMAT0000096 & 4.4196 & CDC34, HMGA2, CDC25A, MAP4K3, CASP3, TTLL4 \\
\hline hsa-let-7d & MIMAT0000065 & 4.6394 & HOXA1, CDC25A, TTLL4, ABCC10 \\
\hline hsa-let-7f & MIMAT0000067 & 4.7517 & CDC25A, HOXA1, HOXA9 \\
\hline hsa-miR-130a & MIMAT0000425 & 4.8953 & PTEN, GAX, HOXA, CSF1, CDC2L11, CDC2L6 \\
\hline hsa-miR-423-5p & MIMAT0004748 & 4.9183 & CASP2, MAP1LC3A, \\
\hline hsa-miR-374a & MIMAT0000727 & 5.5990 & RAB22A, CCNL1 \\
\hline hsa-miRPlus-C1110 & Unknown & 5.8379 & Unknown \\
\hline hsa-miRPlus-A1015 & Unknown & 5.8735 & Unknown \\
\hline hsa-miR-26b & MIMAT0000083 & 5.9615 & CDK6, THAP, PAWR, CCNL2, CDC6, MAP7, MARK1, MAP2, MAP1B \\
\hline hsa-miR-20a & MIMAT0000075 & 6.0910 & EIF5A2, MAP3K2, CDC40, CASP2 \\
\hline hsa-miR-25 & MIMAT0000081 & 6.5038 & CD69, BCL2L11, MOAP1, MARK1 \\
\hline hsa-miR-222 & MIMAT0000279 & 6.8562 & STK17B, CDKN1B, COAS2, CDC2L11, ABCC5 \\
\hline hsa-miR-30e & MIMAT0000692 & 7.2662 & SCN2A, AVEN, CASP3, MAP6, BCL2L11 \\
\hline hsa-miR-320e & MIMAT0015072 & 7.6173 & Unknown \\
\hline hsa-miR-22 & MIMAT0000077 & 7.7164 & TET2, TP53INP1, CDK6, ESR1 \\
\hline hsa-miR-16 & MIMAT0000069 & 8.2800 & BCL2L2, CDC42, MAP7, CDCA4 \\
\hline hsa-miR-374b & MIMAT0004955 & 10.4786 & STK38L, MAP2, ABCE1 \\
\hline hsa-miR-423-3p & MIMAT0001340 & 10.8543 & PABPC1, BCORL1 \\
\hline hsa-miR-29a & MIMAT0000086 & 11.4590 & MARK3, CASP7, MAP6 \\
\hline hsa-miR-19a & MIMAT0000073 & 11.6802 & BCL2L11, ABCA1, CDC2L5, CDC10, MARK2 \\
\hline
\end{tabular}


Table III. Continued.

\begin{tabular}{llcl}
\hline miRNA ID & Accession no. & Fold-change & \multicolumn{1}{c}{ Predicted targets } \\
\hline hsa-miR-20b & MIMAT0001413 & 12.9075 & CDC40, MASTL, CASP2, CASP7, MAPRE3 \\
hsa-miR-331-3p & MIMAT0000760 & 15.1183 & HOXC4, RGS6 \\
hsa-miR-224 & MIMAT0000281 & 16.5871 & API5, HOXD4, CDK9, MAP1B \\
hsa-miR-106b & MIMAT0000680 & 17.7496 & ITGB8, CASP2, EIF5A2 \\
hsa-miR-3607-3p & MIMAT0017985 & 18.5386 & Unknown \\
hsa-miR-15b & MIMAT0000417 & 46.7444 & SCL11A2, CDCA4, MAP7, BCL2L2 \\
\hline
\end{tabular}

Normalized hybridization signal ratio of miRNAs in SKOV3/CIS to those in SKOV3 cells. The normalized fluorescence intensity, which was less than the average intensity of negative controls or 3x SD intensity of negative controls, indicated an unreliable signal value. Differentially expressed miRNAs with statistical significance were selected only if they were altered by at least 2 -fold $(\mathrm{P}<0.05)$. Expression of miRNAs in SKOV3/CIS compared to SKOV3 cells was increased by 2.02- to 46.74-fold. Predicted targets are listed with the help of PicTar and TargetScan.

A-1

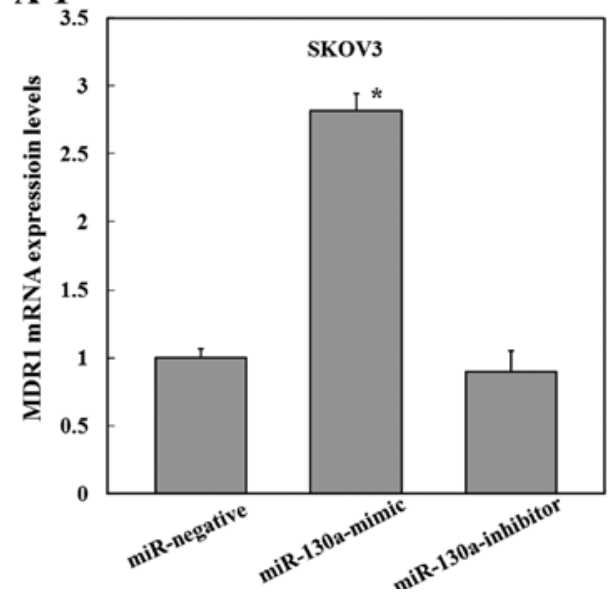

B-1

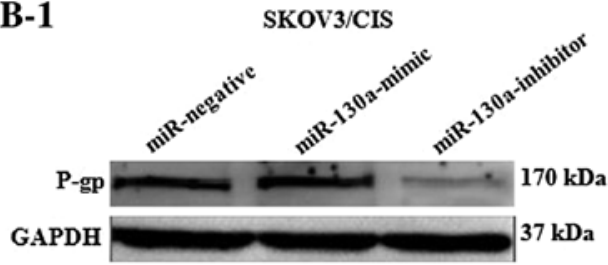

A-2

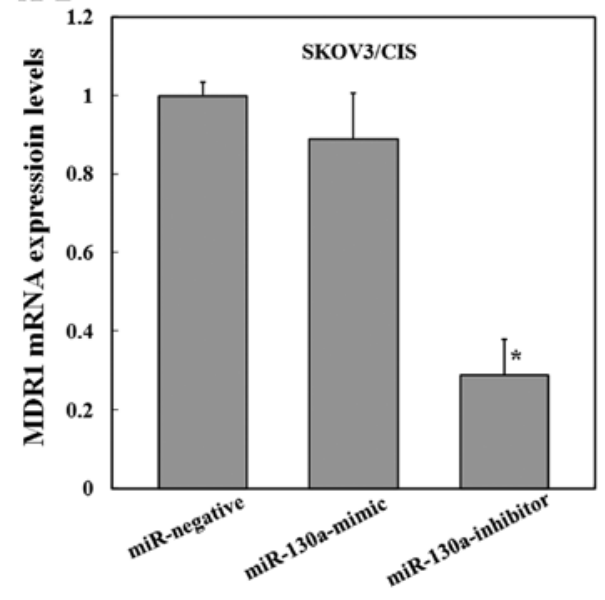

B-2

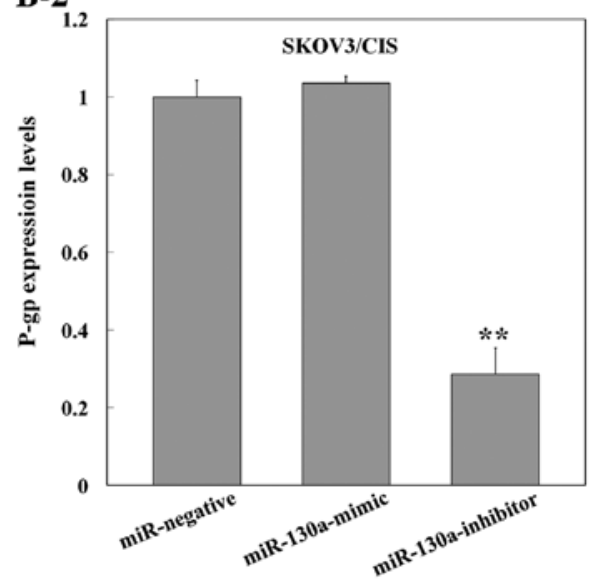

Figure 3. Expression levels of MDR1 mRNA and P-gp in SKOV3 and SKOV3/CIS cells measured by qRT-PCR and western blotting respectively after transfection for $24 \mathrm{~h}$. (A) Expression levels of MDR1 mRNA in SKOV3 and SKOV3/CIS after transfection with a miR-130a-mimic or -inhibitor or with miR-negative. Compared to the miR negative control, the expression of MDR1 mRNA was upregulated in SKOV3 cell transfected with miR-130a-mimic, while it was remarkably decreased in drug-resistant SKOV3/CIS cells transfected with the miR-130a-inhibitor ( ${ }^{*} \mathrm{P}<0.05$, compared to miR-negative control). (B) Expression levels of P-gp in SKOV3/CIS cells after transfection. Transfection with the miR-130a-inhibitor could downregulate P-gp expression in cisplatin-resistant SKOV3/CIS cells $\left({ }^{* *} \mathrm{P}<0.05\right.$ compared to miR-negative control).

miRNAs in developing chemoresistance have been reported. Aberrant levels of miRNAs, including up- and downregulation, might modulate the expression of down-stream target proteins, which could be involved in several molecular mechanisms of drug resistance. For instance, Kovalchuk et al (34) demonstrated that downregulation of miR-451 was associated with doxorubicin resistance in MCF-7 breast cancer cells by regulating P-gp expression. Another study 
A

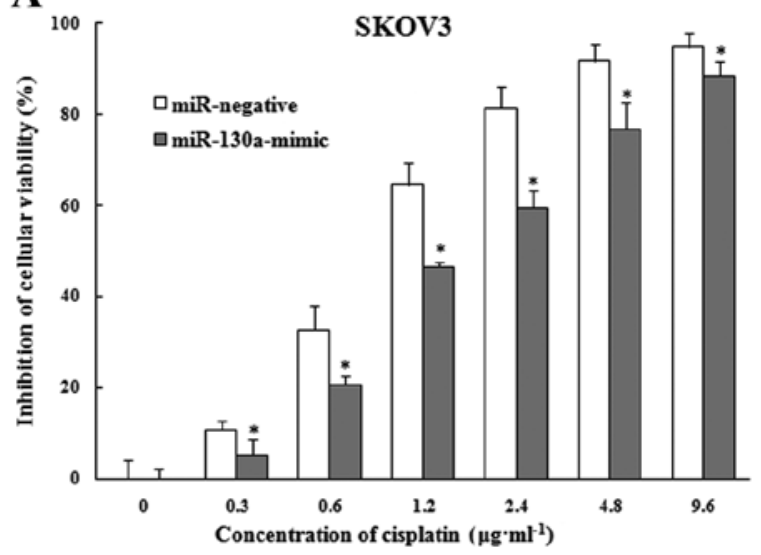

B

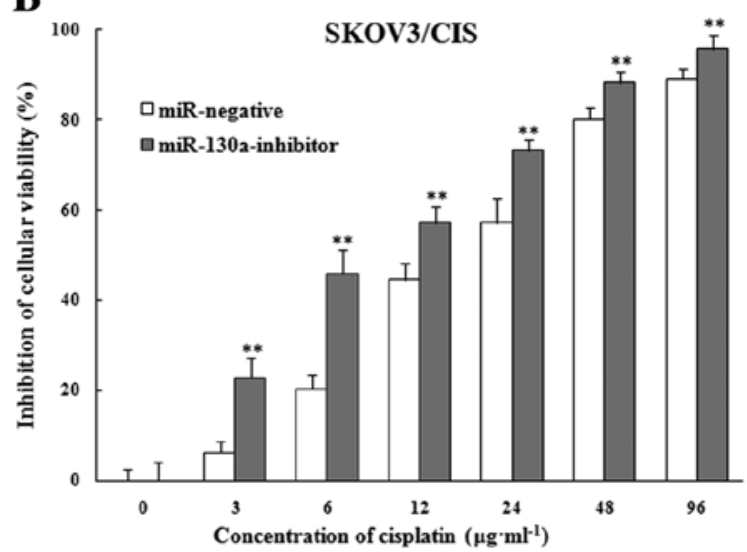

Figure 4. Cisplatin sensitivity is regulated by miR-130a in SKOV3 and SKOV3/CIS cells. (A) SKOV3 cells were transfected with miR-negative and miR-130amimic for $24 \mathrm{~h}$. After transfection, SKOV3 cells were treated with increasing concentrations of cisplatin $(0.3,0.6,1.2,2.4,4.8$ and $9.6 \mu \mathrm{g} / \mathrm{ml})$ for $48 \mathrm{~h}$. Then the cellular viability was detected using the MTT assay. The $\mathrm{IC}_{50}$ of miR-negative and miR-130a-mimic was $0.935 \mu \mathrm{g} / \mathrm{ml} \mathrm{vs} .1 .756 \mu \mathrm{g} / \mathrm{ml}$. (B) SKOV3/CIS cells were transfected with miR-negative and miR-130a-inhibitor for $24 \mathrm{~h}$. After transfection, SKOV3/CIS cells were exposed to increasing doses of cisplatin (3, 6 , $12,24,48$ and $96 \mu \mathrm{g} / \mathrm{ml}$ ) for $48 \mathrm{~h}$. Then the cellular viability was detected using the MTT assay. IC ${ }_{50}$ of miR-negative and miR-130a-inhibitor was $17.402 \mu \mathrm{g} / \mathrm{ml}$ vs. $8.439 \mu \mathrm{g} / \mathrm{ml}$. Columns and bars represented the means and $\mathrm{SE}$ of three independent experiments. $\left({ }^{*} \mathrm{P}<0.05,{ }^{* *} \mathrm{P}<0.05\right.$, compared to the miR-negative control).

reported that $\mathrm{miR}-15 \mathrm{~b}$ and $\mathrm{miR}-16$ could regulate multidrug resistance by targeting BCL-2 in human gastric cancer cells (35). Microarray, a high-throughput analysis, can be used to detect a genome-wide miRNA expression profiling and identify the appropriate candidates of aberrant miRNAs $(36,37)$. Therefore, in the present study with the help of miRNA microarray platform, we have comprehensively isolated and analyzed a total of 1,289 human miRNAs between parental SKOV3 and cisplatin-resistant SKOV3/CIS cells, within which we discovered that 35 miRNAs were downregulated and 54 miRNAs were upregulated in the SKOV3/CIS cell line. Interestingly, some of these miRNAs and their downstream targets were previously reported to be involved in drug resistance of malignant cancer, such as miR-221/-222 targeting p27 (Kip1) in tamoxifen-resistant breast cancer (38); miR-27a targeting MDR1/P-gp in paclitaxel-resistant ovarian cancer (16); and miR-31 targeting E2F6 in prostate cancer cells (39). Consequently, these preliminary results, to the best of our knowledge, suggest that these differentially expressed miRNAs and predicted targeted proteins probably play a crucial role in the development of cisplatin resistance in ovarian cancer, which might provide novel insight for future approaches of gene therapies.

We verified the aberrant expression of miR-130a and performed a further investigation for the function of miR-130a in cisplatin resistance. Here, we found that upregulation of miR130a might be associated with MDR1/P-glycoprotein-mediated drug resistance in SKOV3/CIS cells, while downregulating miR-130a expression might overcome cisplatin resistance. In recent studies, Wang et al (40) have reported that miR-130a expression is increased in non-small cell lung cancer (NSCLC) tissues, which was strongly associated with lymph node metastasis, stage of tumor node metastasis classification and poor prognosis. miR-130a has been reported to be a regulator in antagonizing the inhibitory effects of GAX on endothelial cell proliferation, migration and tube formation, and antagonizing the inhibitory effects of HOXA5 on tube formation in vitro (41). In addition, miR-130a has also been found to be upregulated in MCF-7/ADR breast cancer cells (42). Thereby, we hypothesized that miR-130a acts as an oncogene, which could not only promote angiogenesis, metastasis and growth of cancer cells but also induce multidrug resistance to anticancer chemotherapeutics.

It has been reported that the phosphatidylinositol-3-kinase controlled signal transduction cascade (PI3K/Akt/PTEN/ mTOR pathway) and the activity of drug transporters of the ABC superfamily (P-glycoprotein, MRP1, BCRP) were implicated in resistance of tumor cells to anticancer drugs $(8,9,43)$. With the help of TargetScan, the phosphatase and tensin homolog (PTEN) gene was predicted to be a tumor suppressor and a potential target of miR-130a. Furthermore, our results indicate that miR-130a could modulate the expression levels of MDR1 mRNA and P-gp. A recent study showed that at least two mechanisms of drug resistance are interrelated in prostate cancer cells. PTEN and mTOR signaling were shown to be involved in the regulation of MRP1 and BCRP (44). Consequently, we assumed that miR-130a, acting as an intermediate, might regulate cisplatin resistance by activating PI3K/Akt/PTEN/mTOR and ABC superfamily drug transporter pathways in ovarian cancer cells, which means that different mechanisms involved in drug resistance are probably interconnected.

In summary, we identified the differentially expressed miRNAs in SKOV3 and SKOV3/CIS cells, which suggested that these miRNAs and predicted targeted proteins probably play a crucial role in the development of cisplatin resistance in ovarian cancer. Moreover, we found that upregulation of miR-130a might be associated with MDR1/P-gp-mediated drug resistance in SKOV3/CIS cells and played the role of an intermediate in drug-resistance pathways in PI3K/Akt/PTEN/ mTOR and ABC superfamily drug transporters in ovarian cancer cells. More efforts are needed to further delineate the biofunctional roles of these miRNAs and relevant signaling pathways, which have significant implications in the development of targeted gene therapy for reversing cisplatin resistance in ovarian cancer. 


\section{Acknowledgements}

We are sincerely grateful to Professor Zhao Xia and Manager Yi Tao (Gynecological Oncology of Biotherapy Laboratory, Department of Gynecology and Obstetrics, West China Second University Hospital, Sichuan University) for experimental assistance. This study was supported by the Program for Changjiang Scholars and the Innovative Research Team (PCSIRT) in University (IRT0935) and the local Foundation of Science and Technology Department of Sichuan Province (no. 2009S20161).

\section{References}

1. Legge F, Ferrandina G, Salutari V, et al: Biological characterization of ovarian cancer: prognostic and therapeutic implications. Ann Oncol 16: 95-101, 2005.

2. Heintz AP, Odicino F, Maisonneuve P, et al: Carcinoma of the ovary. FIGO 6th Annual Report on the Results of Treatment in Gynecological Cancer. Int J Gynaecol Obstet 95: S161-S192, 2006.

3. Du Bois A and Pfisterer J: Future options for first-line therapy of advanced ovarian cancer. Int J Gynecol Cancer 15: 42-50, 2005.

4. Foster T, Brown TM, Chang J, et al: A review of the current evidence for maintenance therapy in ovarian cancer. Gynecol Oncol 115: 290-301, 2009.

5. de Jongh FE, de Wit R, Verweij J, et al: Dose-dense cisplatin/ paclitaxel: a well-tolerated and highly effective chemotherapeutic regimen in patients with advanced ovarian cancer. Eur J Cancer 38: 2005-2013, 2002.

6. Muggia F: Platinum compounds 30 years after the introduction of cisplatin: implications for the treatment of ovarian cancer. Gynecol Oncol 112: 275-281, 2009.

7. Rabik CA and Dolan ME: Molecular mechanisms of resistance and toxicity associated with platinating agents. Cancer Treat Rev 33: 9-23, 2007.

8. Hu M, Liu Y, Deng C, Han R, et al: Enhanced invasiveness in multidrug resistant leukemic cells is associated with overexpression of P-glycoprotein and cellular inhibitor of apoptosis protein. Leuk Lymphoma 52: 1302-1311, 2011.

9. Gottesman MM and Ling V: The molecular basis of multidrug resistance in cancer: the early years of P-glycoprotein research. FEBS Lett 580: 998-1009, 2006.

10. Park E, Gang EJ, Hsieh YT, et al: Targeting survivin overcomes drug resistance in acute lymphoblastic leukemia. Blood 118 2191-2199, 2011.

11. Fraser M, Bai T and Tsang BK: Akt promotes cisplatin resistance in human ovarian cancer cells through inhibition of p53 phosphorylation and nuclear function. Int J Cancer 122: 534-546, 2008 .

12. Cheung $\mathrm{CH}, \mathrm{Wu} \mathrm{SY}$, Lee TR, et al: Cancer cells acquire mitotic drug resistance properties through beta I-tubulin mutations and alterations in the expression of beta-tubulin isotypes. PLoS One 5: e12564, 2010

13. Zindy P, Bergé Y, Allal B, et al: Formation of the eIF4F translation-initiation complex determines sensitivity to anticancer drugs targeting the EGFR and HER2 receptors. Cancer Res 71: 4068-4073, 2011.

14. Sabatino MA, Marabese M, Ganzinelli M, et al: Down-regulation of the nucleotide excision repair gene XPG as a new mechanism of drug resistance in human and murine cancer cells. Mol Cancer 9: 259,2010

15. Kirschner K and Melton DW: Multiple roles of the ERCC1-XPF endonuclease in DNA repair and resistance to anticancer drugs. Anticancer Res 30: 3223-3232, 2010.

16. Li Z, Hu S, Wang J, Cai J, et al: MiR-27a modulates MDR1/Pglycoprotein expression by targeting HIPK2 in human ovarian cancer cells. Gynecol Oncol 119: 125-130, 2010.

17. Bartel DP: MicroRNAs: genomics, biogenesis, mechanism, and function. Cell 116: 281-297, 2004.

18. Denli AM, Tops BB, Plasterk RH, et al: Processing of primary microRNAs by the Microprocessor complex. Nature 432: $231-235,2004$
19. Berezikov E, Guryev V, van de Belt J, et al: Phylogenetic shadowing and computational identification of human microRNA genes. Cell 120: 21-24, 2005.

20. Lund E, Güttinger S, Calado A, et al: Nuclear export of microRNA precursors. Science 303: 95-98, 2004.

21. He L and Hannon GJ: MicroRNAs: small RNAs with a big role in gene regulation. Nat Rev Genet 5: 522-531, 2004.

22. Kloosterman WP and Plasterk RH: The diverse functions of microRNAs in animal development and disease. Dev Cell 11: 441-450, 2006.

23. Esquela-Kerscher A and Slack FJ: Oncomirs-microRNAs with a role in cancer. Nat Rev Cancer 6: 259-269, 2006.

24. Iorio MV, Visone R, Di Leva G, et al: MicroRNA signatures in human ovarian cancer. Cancer Res 67: 8699-8707, 2007.

25. Yanaihara N, Caplen N, Bowman E, et al: Unique microRNA molecular profiles in lung cancer diagnosis and prognosis. Cancer Cell 9: 189-198, 2006.

26. Calin GA, Dumitru CD, Shimizu M, et al: Frequent deletions and down-regulation of microRNA genes miR15 and miR16 at 13q14 in chronic lymphocytic leukemia. Proc Natl Acad Sci USA 99: $15524-15529,2002$.

27. Gong C, Yao Y, Wang Y, et al: Up-regulation of miR-21 mediates resistance to trastuzumab therapy for breast cancer. J Biol Chem 286: 19127-19137, 2011.

28. Hwang JH, Voortman J, Giovannetti E, et al: Identification of microRNA-21 as a biomarker for chemoresistance and clinical outcome following adjuvant therapy in resectable pancreatic cancer. PLoS One 5: e10630, 2010

29. Tomimaru Y, Eguchi H, Nagano H, et al: MicroRNA-21 induces resistance to the anti-tumour effect of interferon- $\alpha / 5$-fluorouracil in hepatocellular carcinoma cells. Br J Cancer 103: 1617-1626, 2010.

30. Krek A, Grün D, Poy MN, et al: Combinatorial microRNA target predictions. Nat Genet 37: 495-500, 2005.

31. Grün D, Wang YL, Langenberger D, et al: microRNA target predictions across seven Drosophila species and comparison to mammalian targets. PLoS Comput Biol 1: e13, 2005.

32. Lewis BP, Shih IH, Jones-Rhoades MW, et al: Prediction of mammalian microRNA targets. Cell 115: 787-798, 2003.

33. Lewis BP, Burge CB and Bartel DP: Conserved seed pairing, often flanked by adenosines, indicates that thousands of human genes are microRNA targets. Cell 120: 15-20, 2005.

34. Kovalchuk O, Filkowski J, Meservy J, et al: Involvement of microRNA-451 in resistance of the MCF-7 breast cancer cells to chemotherapeutic drug doxorubicin. Mol Cancer Ther 7: 2152-2159, 2008.

35. Xia L, Zhang D, Du R, et al: MiR-15b and miR-16 modulate multidrug resistance by targeting BCL2 in human gastric cancer cells. Int J Cancer 123: 372-379, 2008.

36. Lu J, Getz G, Miska EA, et al: MicroRNA expression profiles classify human cancers. Nature 435: 834-838, 2005.

37. Pallante P, Visone R, Ferracin M, et al: MicroRNA deregulation in human thyroid papillary carcinomas. Endocr Relat Cancer 13: 497-508, 2006.

38. Miller TE, Ghoshal K, Ramaswamy B, et al: MicroRNA-221/222 confers tamoxifen resistance in breast cancer by targeting p27Kip1. J Biol Chem 283: 29897-29903, 2008

39. Bhatnagar N, Li X, Padi SK, et al: Downregulation of miR-205 and miR-31 confers resistance to chemotherapy-induced apoptosis in prostate cancer cells. Cell Death Dis 1: e105, 2010.

40. Wang XC, Tian LL, Wu HL, et al: Expression of miRNA-130a in nonsmall cell lung cancer. Am J Med Sci 340: 385-388, 2010.

41. Chen Y and Gorski DH: Regulation of angiogenesis through a microRNA (miR-130a) that down-regulates antiangiogenic homeobox genes GAX and HOXA5. Blood 111: 1217-1226, 2008.

42. Cui XY, Guo YJ and Yao HR: Analysis of microRNA in drugresistant breast cancer cell line MCF-7/ADR. Nan Fang Yi Ke Da Xue Xue Bao 28: 1813-1815, 2008 (In Chinese).

43. Naumann RW: The role of the phosphatidylinositol 3-kinase (PI3K) pathway in the development and treatment of uterine cancer. Gynecol Oncol 123: 411-420, 2011.

44. Sherbakova EA, Stromskaia TP, Rybalkina EIu, et al: Role of PTEN protein in multidrug resistance of prostate cancer cells. Mol Biol (Mosk) 42: 487-493, 2008 (In Russian). 